\title{
Brain Image Segmentation Using Multistable Cellular Neural Networks
}

\author{
Burak Yılmaz $^{1 *}$, Akif Durdu, Güzin Özmen ${ }^{2}$ \\ ${ }^{1}$ Konya Food and Argiculture University, Turkey \\ ${ }^{2}$ Selcuk Unversity Faculty of Enginnering Electrics-Electronics Engineering Department Konya, Turkey. \\ * Corresponding author. Tel.: +90 53363486 38; email: burak.yilmaz@gidatarim.edu.tr \\ Manuscript submitted May 20, 2017; accepted July 20, 2017. \\ doi: $10.17706 /$ jcp.13.5.
}

\begin{abstract}
This study proposes a new method on "brain image segmentation". Algorithm is based on the Multistable Cellular Neural Networks (Multistable-CNN). Evaluations are performed on well-known BRAINWEB database. The metrics for performance evaluations of the algorithm were JACCARD, DICE, TPF and TNF. The results are compared with the SPM8, FSL FAST and Brain Suite software packages. The Multistable CNN algorithm used in this study differs from conventional CNN algorithm. The Multistable CNN's can perform multiple segmentations in a single run. Algorithm is simple and doesn't require complex calculations. Evaluations show that proposed algorithm's performance is adequate. Algorithm also does not require any atlas or image registration
\end{abstract}

Key words: MRI, brain, segmentation, cellular neural networks, multistable cellular neural networks, cerebrospinal fluid (CSF), gray matter (GM) and white matter (WM).

\section{Introduction}

Medical imaging techniques have a very common use in the anatomical and medical studies. Due to its non-invasive and non-destructive nature Magnetic Resonance Imaging (MRI) technique is generally preferred [1]. The MRI images need intense analysis, depending on the studies to be performed. Hence before performing these studies, images needed to be preprocessed and segmented into different regions. A typical brain MRI image consists of following regions; background, fat, muscle, skin, skull, vessels, marrow, cerebrospinal fluid (CSF), gray matter (GM) and white matter (WM).

There are many brain image segmentation algorithms exist in the literature. These algorithms based on different methods such as, contour, shape and region based, statistical, probability, multiresolution, atlas, pattern recognition, machine learning and hybrid methods[2].

There are some well known software packages are also exists for neuroimaging analysis[3]. Most known packages can be listed as, SPM8 toolbox which is developed at Wellcome Trust Centre for Neuroimaging, (University College London, UK), FSL software package written by Analysis Group, (FMRIB Oxford, UK) and BrainSuite developed by Dr. David W. Shattuck [3].

In this study, a new approach based on multistable cellular neural networks (MS-CNN) for segmenting the CSF, GM and WM regions in the brain is used. The MS-CNN templates and the polynomial coefficients are generated by the artificial bee colony algorithm ( $\mathrm{ABC}$ ) proposed by Karaboğa et al. [4]

Before the segmentation, some image processing techniques are generally used for preprocessing for improving success rates. In this study, standard average filtering is used for preprocessing.

The algorithm is evaluated with BRAINWEB data set. The dataset consists of 20 T1 weighted MRI images 
which also have ground truth images within. Evaluations performed with the following metrics; Jaccard, Dice, True Positive Fraction (TPF) and True Negative Fraction (TNF) parameters.

The algorithm also compared with the SPM8, FSL FAST and Brain Suite software packages which are previously evaluated by Kasiri et al. [3]

\section{Methodology}

\subsection{Multistable Cellular Neural Networks}

Cellular Neural Networks (CNNs) are two dimensional cell arrays with local cell interconnections. Chua and Yang introduced CNNs in 1998 [5], [6]. Every cell is related with the neighboring cells presented in Fig 1. [5], [6]. The CNNs differ from the conventional Neural Networks with the local connectivity property. Because of this property CNNs are able to perform image processing and pattern recognition operations [7], [8]. In Fig. 1 cell structure of a CNN is shown [5], [6].



Fig. 1. CNN cell structure.

Neighborhood level of each cell is defined by the constant ' $r$ ' where $r=1$ means $3 \times 3$ neighborhood, $r=2$ means $5 x 5$ neighborhood and so on. This is called sphere of influence and $r$ means the radius of the sphere [9]

Each CNN cell is described with a normalized differential state equation (1).

$$
\frac{d}{d x} x_{i, j}(t)=\sum_{k, l \in N} A_{k l} y_{i+k, j+k}(t)+\sum_{k, l \in N} B_{k l} y_{i+k, j+k}(t)+I
$$

Nonlinearity of output is defined by Eq. (2), this equation represents a trapezoidal function limited between $(-1,+1) \in \mathbb{Q}$ and used as output of the CNN

$$
y(x)=\frac{1}{2}[|x-1|-|x+1|]
$$

The input, state, and the output of the CNN, are represented as $u_{i, j}, x_{i, j}$ and $y_{i, j}$ respectively. The $i, j$ values are defined in $0 \leq i \leq N_{1}$ and $0 \leq j \leq N_{2}$. In the equation (1) $N_{1}$ and $N_{2}$ represents length and width of the input image. In the equation (2) $x$ is the same as equation (1).

There is an advanced model of CNN's exists in the literature, which is named as the Multistable CNNs. The Multistable CNNs differ from the conventional CNNs with the definition of their output functions. In the conventional CNN model, algorithm approximates to final state in every iteration, the output function have one slope and limited between $(-1,+1) \in \mathbb{Q}$. When the CNN reaches to the steady state algorithm finishes 
classification of the pattern, ( 1 for member, -1 for non-member). In the Multistable CNNs cell definitions are same with the conventional CNN cell, but the output function is defined as polynomial functions of different ranks or piecewise nonlinear functions with more slopes instead of unit saturation function [10] which makes the multistable CNN capable of classifying more than two classes, In this study a polynomial function is preferred for the multistable cellular neural network.

In the MS-CNN model defining a proper output function is very important task. There are some rules must be followed for a proper output function definition. These basic rules are as follows; the function must be continuous, it must have real roots and it must be symmetrical.

Considering the rules, the possible output function can be designed as the following polynomial function:

$$
f(x)=a\left(x-r_{1}\right)\left(x-r_{2}\right) \ldots\left(x-r_{n}\right)\left(x-r_{n+1}\right) \ldots\left(x-r_{2 n+1}\right)
$$

where $\mathrm{a}<0$ and $r_{1}<r_{2}<\ldots<r_{2 n+1}$ are real numbers, $r_{1}, r_{2} \ldots, r_{n}$ are negative $r_{n+1}=0$ and $r_{n+2}, r_{n+3} \ldots, r_{2 n+1}$ are positive [11]. Function is defined in factor form which gives an opportunity to find the ' $r$ ' coefficients with optimization algorithms. Factor form is a generalized form for representing polynomials. The multistable CNN model used in the algorithm is represented in Fig. 2.

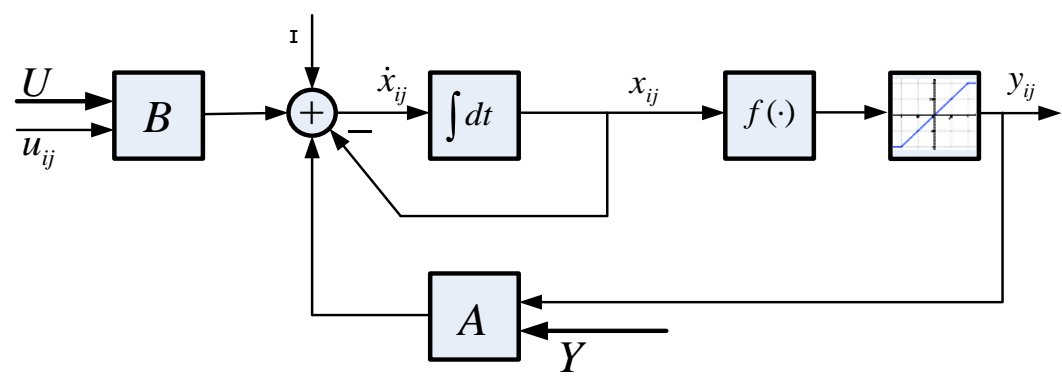

Fig. 2. Multistable CNN cell model.

In this model, the input is given as $\mathrm{U}$ and $\mathrm{Y}$ represents initial condition. Here $u_{i j}$ represents input from the neighboring cells and $x_{i j}$ represents the initial condition from the neighboring cells. CNNs work with three specific sets of parameters which called templates. Each cell has a matrix template which is represented with the triplet $\{A, B, I\}$. The matrix $A$ represents all of the $A_{k l}$ values, and the matrix $B$ represents the all of the $B_{k l}$ values. There are some rules should be considered while defining the $A$ and $B$ matrices. The matrices must be square matrices like $3 \times 3,5 \times 5$ and etc. The threshold I is generally defined as a one-dimensional $1 \times 1$ matrix but it can a, $\left[r_{1}, r_{2}, r_{3} \ldots, r_{n}\right]$ lso be defined a matrix with the same size of $A$ and $B$ matrices [10]. Determining the values of template matrices is called template learning. Values of the matrices affects the general dynamics of the CNN cell, hence the training creates a stable connection network for the task to be worked on. In this study the artificial bee colony (ABC) algorithm developed by Karaboğa et al. [4] is used for template learning task.

The $a,\left[r_{1}, r_{2}, r_{3} \ldots, r_{n}\right]$ parameters of the output function determined with the ABC algorithm.

\subsection{The Artificial Bee Colony Algorithm and Template Learning}

The ABC algorithm can be summarized as an algorithm that simulates the food search task in a bee 
colony. Algorithm simulates swarm behaviors of a bee colony [4]. The algorithm evaluates the success of the function with values found after each search phase. $A$ fitness function is required for this evaluation step. The fitness function is always designed specifically for the problem.

In this study in the training step 2D image correlation function is used as the fitness function. Function is explained in equation (4).

$$
r=\frac{\sum_{m} \sum_{n}\left(A_{m n}-\dot{A}\right)\left(B_{m n}-\dot{B}\right)}{\sqrt{\left(\sum_{m} \sum_{n}\left(A_{m n}-\ddot{A}\right)^{2}\right)\left(\sum_{m} \sum_{n}\left(B_{m n}-\dot{B}\right)^{2}\right)}}
$$

In this formula $A$ and $B$ are images to be compared and $\vec{A}$ is 2D mean value of image $A, B$ is 2D mean value of image $B$.

The correlation coefficient is between [0 - 1] and values closer to 1 means successful segmentation. In this study values between [0.86 - 1] are considered as acceptable values, based on experience.

In this study templates are represented in vector form for compatibility with the ABC algorithm[9]. Vectorization of the templates matrices is shown in the Fig. 3.

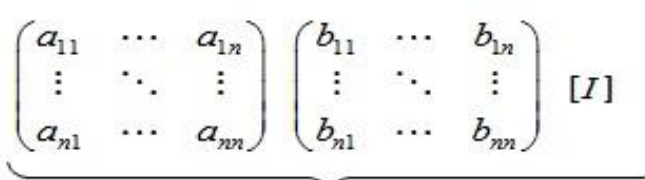

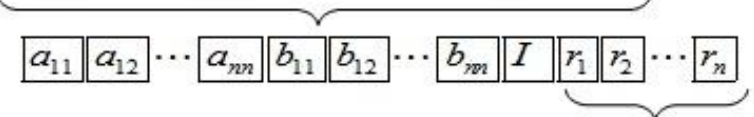

$$
\begin{aligned}
& \begin{array}{l}
\text { Roots of output } \\
\text { function }
\end{array}
\end{aligned}
$$

Fig. 3. Vectorization of the CNN templates.

After the template learning process the template matrices and polynomial coefficients are determined as follows:

$$
\begin{aligned}
A & =\left[\begin{array}{lll}
-0,38 & 1 & -0.46 \\
0.195 & -1 & 0.195 \\
-0.21 & 0.72 & -0.18
\end{array}\right], B=\left[\begin{array}{lll}
-0.51 & -0.17 & 0.67 \\
-0.95 & 0.57 & 0.5 \\
-0.21 & -0.44 & 0.96
\end{array}\right], I=[0.45] \\
r & =\left[\begin{array}{lll}
0.93 & 0.65 & 0.18
\end{array}\right]
\end{aligned}
$$

The general flowchart of the algorithm is shown in Fig. 4.



Fig. 4. General flowchart of the algorithm. 
Input and output images of algorithm is shown in Fig. 5 source image is, slice 120 of the subject04 in the BRAINWEB database.

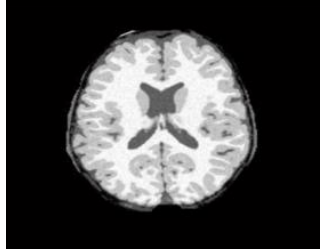

(a)

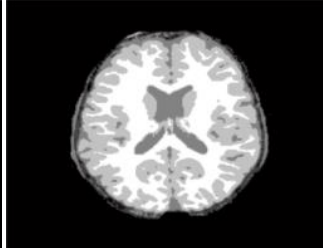

(b)



(c)

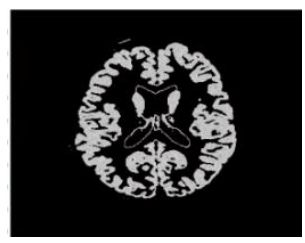

(d)

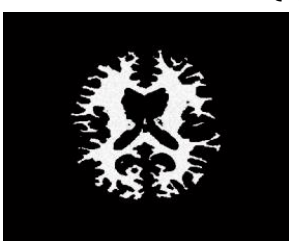

(e)

Fig. 5. Output images of algorithm (a) input image (b) Output of multistable CNN (c) pixels labeled as CSF with the algorithm CNN (d) pixels labeled as GM with the algorithm CNN (e) pixels labeled as WM with the algorithm.

\section{Performance Evaluation}

\subsection{Brainweb Dataset}

The Brainweb database is a 3-dimensional database with various modalities provided by the McConnell Brain Imaging Center of the Montreal Neurological Institute of McGill University. Brainweb provides 20 anatomical models of normal brain MRI images[12], [13]. The original images in the database have the following properties; $1 \mathrm{~mm}$ slice thickness, T1 weighted, $181 \times 256 \times 256$ voxels with signed short integer values between 0 and 4095. Every original image also have an anatomical model with the following properties $0.5 \mathrm{~mm}$ slice thickness, $362 \times 434 \times 362$ voxels with signed integer values between 0 and 11 . Original images are skull stripped with FSL program[14] and anatomical model is used as ground truth.

\subsection{Evaluation Parameters}

In the evaluation process algorithm generated digital masks of every segmented regions. Each region classified as integer numbers by the algorithm. The class number values are 252 and 253 for CSF, 254 for GM and 255 for WM. Quantitative evaluations performed with the commonly used JACCARD, DICE, TPF, TNF parameters. JACCARD and DICE coefficients represents overlap between two binary images, values range between 0 and 1 which " 0 " represents no overlaps and " 1 " represents full match. Definitions of these metrics are as follows:

$$
\begin{gathered}
D=\frac{2|S \cap R|}{|S|+|R|} \times 100 \\
J=\frac{|S \cap R|}{S \cup R} \times 100=\frac{D}{2-D}
\end{gathered}
$$

TPF, TNF parameters represents sensitivity and specificity and they are defined as follows: 


$$
\begin{aligned}
& T P F=\frac{T P}{T P+F N} \\
& T N F=\frac{T N}{T N+F P}
\end{aligned}
$$

which $S$ represents result of the segmentation algorithm and $R$ represents ground truth obtained from database.

\subsection{Evaluation Results}

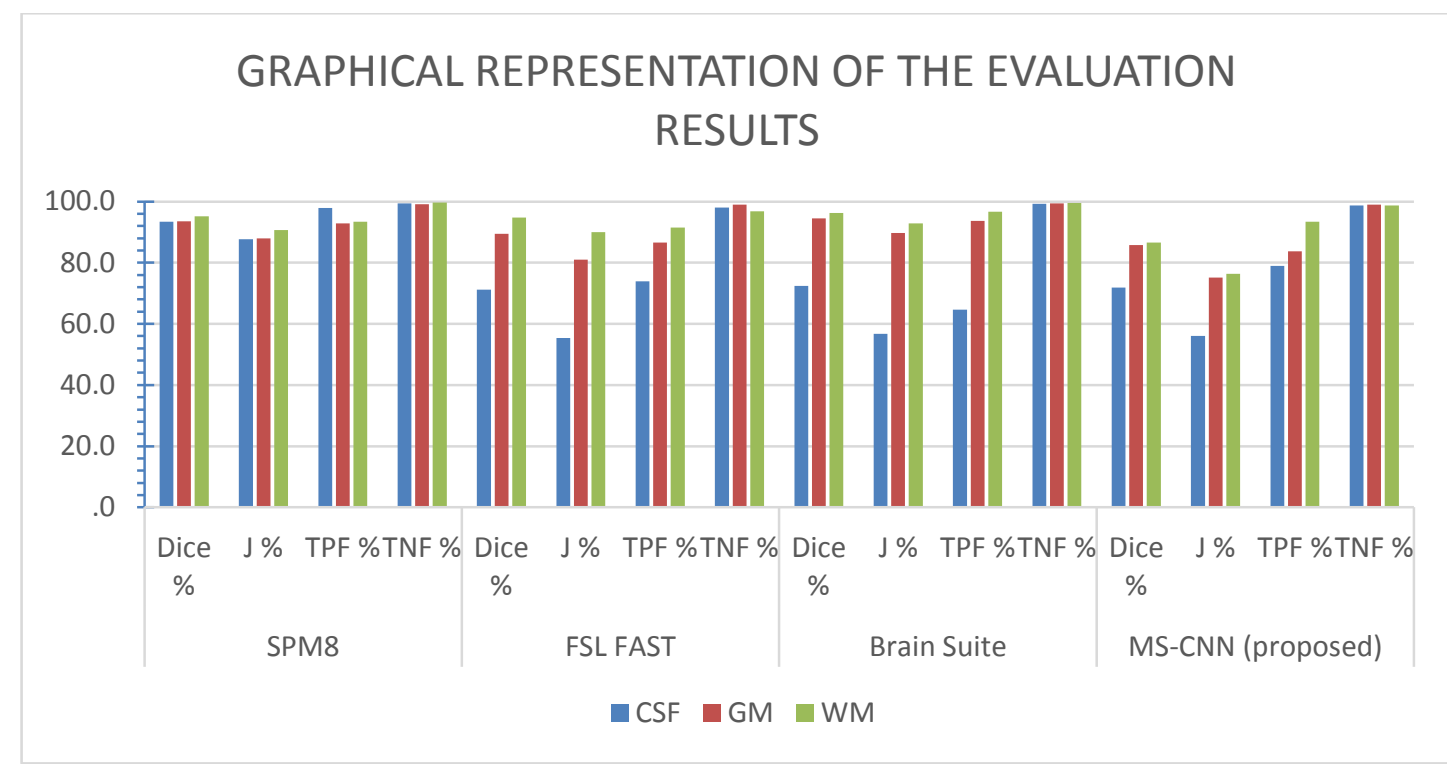

Fig. 6. Graphical representation of the evaluation results.

Table 1. Evaluation Results Compared with the SPM8, FSL FAST and Brain Suite Software Packages [3]

\begin{tabular}{llllllll}
\hline \hline \multirow{2}{*}{ Method } & Metric & \multicolumn{2}{l}{ CSF } & & GM & & WM \\
\cline { 2 - 7 } & & Mean & STD & Mean & STD & Mean & STD \\
\hline SPM8 & Dice \% & 93.39 & 0.56 & 93.55 & 0.7 & 95.12 & 0.95 \\
& J \% & 87.6 & 0.99 & 87.89 & 1.24 & 90.7 & 1.72 \\
& TPF \% & 97.91 & 0.52 & 92.81 & 1.63 & 93.37 & 2.96 \\
& TNF \% & 99.33 & 0.05 & 99.18 & 0.31 & 99.69 & 0.2 \\
FSL FAST & Dice \% & 71.22 & 1.98 & 89.46 & 0.63 & 94.70 & 1.39 \\
& J \% & 55.33 & 2.39 & 80.93 & 1.03 & 89.95 & 2.49 \\
& TPF \% & 73.92 & 3.89 & 86.54 & 1.36 & 91.53 & 3.77 \\
Brain Suite & TNF \% & 98.09 & 0.07 & 98.99 & 0.42 & 96.82 & 0.17 \\
& Dice \% & 72.37 & 0.35 & 94.52 & 1.46 & 96.23 & 1.52 \\
& J \% & 56.70 & 0.43 & 89.65 & 2.63 & 92.78 & 2.84 \\
MS-CNN & TPF \% & 64.58 & 0.43 & 93.60 & 1.87 & 96.60 & 0.95 \\
(proposed) & TNF \% & 99.21 & 0.04 & 99.35 & 0.15 & 99.56 & 0.23 \\
& Dice \% & $\mathbf{7 1 . 8 0}$ & $\mathbf{2 . 3 6}$ & $\mathbf{8 5 . 7 4}$ & $\mathbf{1 . 2 6}$ & $\mathbf{8 6 . 6 2}$ & $\mathbf{1 . 0 6}$
\end{tabular}


The evaluation was performed on 20 normal T1-weighted MRI images obtained from Brainweb database. Images are presented as initial condition and a matrix consists of zeros as presented as input to multistable CNN algorithm. Every subject segmented into 3 regions, which are GM, WM and CSF. Graphical representation of the evaluation results are shown in the Fig. 6.

Segmentation performance evaluation values with the standard deviation values are also shown as in the Table 1. The proposed algorithm is compared with the SPM8, FSL FAST and Brain Suite software packages [3]. SPM8, FSL FAST and Brain Suite software packages has previously been examined in the ref. [3], the Table1 includes values obtained from the ref. [3] and the evaluation results of the proposed algorithm both.

\section{Conclusion}

In this study, a new technique using Multistable CNN for brain image segmentation is shown. Technique has a unique advantage, which is Multistable CNN algorithm is capable of performing multiple segmentations in a single run. Evaluations show that proposed algorithm's performance not lagging behind the others. It can easily be said that SPM8 program is best amongst the other algorithms, because SPM8 performs not only pure image processing but also statistical analysis too. This makes SPM8 algorithm more precise. On the other hand the difference between MS-CNN algorithm and the other algorithms do not have great values, hence it can be said that this primitive version of MS-CNN algorithm, is promising.

The algorithm has also some extra capabilities like working on non-Talairach-compatible data because algorithm does not require any atlas or image registration. Algorithm works slice by slice manner and analyzes each slice independently, hence algorithm can perform parallel analysis in multi-core processors.

On the other hand, the poor performance of the algorithm is because of the algorithm can't smoothen the boundaries between the classes. In the future studies, new steps will be added for handling this issues. It can be said that, the algorithm's pattern detection ability is promising, in the future studies, increasing the precision of the algorithm is aimed.

\section{References}

[1] Somasundaram, K., \& Kalaiselvi, T. (2011). Automatic brain extraction methods for T1 magnetic resonance images using region labeling and morphological operations. Computers in Biology and Medicine, 41, 716-725.

[2] El-Dahshan, E. A., Mohsen, H. M., Revett, K., \& Salem, A. B. M. (2014). Computer-aided diagnosis of human brain tumor through MRI: A survey and a new algorithm. Expert Systems with Applications, 41, 5526-5545.

[3] Kasiri, K., Javad, D. M., Kazemi, K., Sadegh, H. M., \& Kafshgari, S. (2010). Comparison evaluation of three brain MRI segmentation methods in software tools. Proceedings of the 17th Iranian Conference on Biomedical Engineering (ICBME) (pp. 1-4).

[4] Karaboga, D., Gorkemli, B., Ozturk, C., \& Karaboga, N. (2012). A comprehensive survey: Artificial bee colony $(\mathrm{ABC})$ algorithm and applications. Artificial Intelligence Review.

[5] Chua, L. O., \& Yang, L. (1988). Cellular neural networks: Theory. IEEE Transactions on Circuits and Systems, 35, 1257-1272.

[6] Chua, L. O., \& Yang, L. (1988). Cellular neural networks: Applications. IEEE Transactions on Circuits and Systems, 35.

[7] Kawahara, M., Inoue, T., \& Nishio, Y. (2009). Cellular neural network with dynamic template and its output characteristics, Proceedings of the International Joint Conference on Neural Networks (pp. 1552-1558).

[8] Perfetti, R., Ricci, E., Casali, D., \& Costantini, G. (2007). Cellular neural networks with virtual template 
expansion for retinal vessel segmentation. IEEE Transactions on Circuits and Systems II: Express Briefs, 54, 141-145.

[9] Cerasa, A., Bilotta, E., Augimeri, A., Cherubini, A. (2012). A cellular neural network methodology for the automated segmentation of multiple sclerosis lesions. Journal of Neuroscience Methods, 203(1), 193-199.

[10] Yokosawa, K., Nakaguchi, T., Tanji, Y., \& Tanaka, M. (2003). Cellular neural networks with output function having multiple constant regions. IEEE Transactions on Circuits and Systems I: Fundamental Theory and Applications, 50, 847-857.

[11] Hernandez, J. A. M., Castaeda, F. G., \& Cadenas, J. A. M. (2009). Multistable cellular neural networks and their application to image decomposition. Proceedings of the 52nd IEEE International Midwest Symposium on Circuits and Systems.

[12] Aubert, B., Evans, A. C., \& Collins, L. (2006). A new improved version of the realistic digital brain phantom. NeuroImage, 32(1), 138-145.

[13] Aubert, B., Griffin, M., Pike, G. B., Evans, A. C., \& Collins, D. L. (2006). Twenty new digital brain phantoms for creation of validation image data bases. IEEE Transactions on Medical Imaging, 25, 1410-1416.

[14] Jenkinson, M., Beckmann, C. F., Behrens, T. E. J., Woolrich, M. W., \& Smith, S. M. (2012). Fsl.' NeuroImage, $62(2), 782-790$.



Burak Yılmaz has been a research specialist with the IT Department at the Selcuk University (SU) since 2010. He earned the masters degree in computer engineering from the Selcuk University (S.U.), in 2007. He received his B.Sc. degree in eletrical-electronics engineering in 2001 at the Firat University. His research interests include image processing, biomedical image analyzing, pattern recognition.

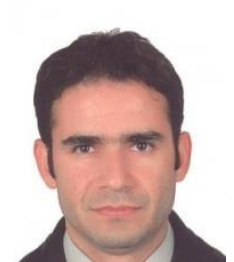

Akif Durdu has been an assistant professor with the Eletrical-Electronics Engineering Department at the Selcuk University (SU) since 2013. He earned the Ph.D. degree in eletrical-electronics engineering from the Middle East Technical University (METU), in 2012. He received his B.Sc. degree in eletrical-electronics engineering in 2001 at the Selcuk University. His research interests include mechatronic design, search and rescue robotics, robot manipulators, human-robot interaction, multi-robots networks and sensor networks. Durdu is teaching courses in control engineering, robotic and mechatronics systems.

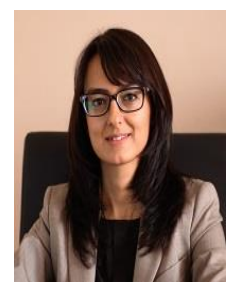

Güzin Özmen has been a lecturer with the Eletrical-Electronics Engineering Department at Technology Faculty at the Selcuk University (SU) since 2016. She received the masters degree eletrical-electronics engineering from the Selcuk University (S.U.) in 2013. She received her B.Sc. degree in eletrical-electronics engineering in 2006 at the Selcuk University. Her research interests include image processing, signal processing, biomedical image analyzing. 